\title{
CAN TWO SOLIDS SLIDE WITHOUT SLIPPING?
}

\author{
Maria Comninou \\ University of Michigan, Ann Arbor, MI 48109, U.S.A. \\ and \\ J. Dundurs \\ Northwestern University, Evanston, IL 60201. U.S.A. \\ (Receiced 12 May 1977: retised 15 July 1977: receiced for publication I September 1977)
}

\begin{abstract}
The question posed is whether two solids that are pressed together and at the same time sheared can undergo a sliding motion without slip at the interface. The mechanism involves a propagating separation zone, with the tangential shift between the solids taking place in this zone whilst they are out of contact. It is shown that the applied forces can sustain such motion, and that the separation zones can propagate with velocities above the speed of surface waves and also in the transonic range. If such motion is induced, sliding can occur even at very low levels of applied shearing forces.
\end{abstract}

\section{INTRODUCTION}

A rug can be moved from one place on the floor to another by pulling along one of its edges until friction is overcome and simply making it slip. As is known to every tradesman who has to handle large pieces of carpeting, which are not easily moved by pulling, there is another way that takes much less effort. The carpet can be given a fold at one end, and the fold made to propagate in the direction in which the carpet has to be moved. This process does not require that friction be overcome and involves no slip. Moreover, the carpet can be given any apparent sliding motion with respect to the floor by propagating a series of folds.

The carpet fold is often cited in dislocation theory[1]. A dislocation in a crystal lattice provides an escape mechanism through which plastic deformations can take place more easily than by slipping a whole atomic plane over another. Similarly, the propagating carpet fold leads to a global change that requires only a local readjustment and little expenditure of work by an external agent. The question we investigate is whether the "carpet fold" is a feasible escape mechanism which leads to an overall sliding motion between two solids that are pressed together and at the same time sheared, but does not involve local slip. We do not enquire how the motion gets started, but merely explore whether it can possibly be sustained by the applied forces.

Motions of a related nature have been observed in sliding between rubber and glass. They are known now as Schallamach waves. First reported in 1971[2], these waves involve zones of local detachment between the two materials. The relative tangential displacement takes place in the separation zones whilst the bodies are out of contact, and there is little, if any local slip in the zones where the bodies are in contact. Several confirming observations have been described since, and propagating zones of separation have also been observed in pulling stiff fibers out of a soft matrix by Kendall[3], who calls them bubble cracks (if interested, the reader can easily trace the pertinent articles using Schallamach's paper [2] as entry in the Citation Index). The Schallamach waves are very slow in comparison to elastic interface waves, which indicates that they are not controlled by inertia. Indeed, Barquins and Courtel[4] conclude that the Schallamach waves of detachment are governed by relaxation effects and adhesion between the two materials.

In contrast, our investigation is concerned with a process that is controlled by elasticity and inertia. The problem is considered in the context of elasticity theory and, for simplicity, only identical materials are studied. The formulation takes advantage of the known stress fields for moving edge dislocations, and the essential aspects of the problem are quickly reduced to singular integral equations which have closed form solutions. The conclusion we reach is that the anticipated motion can be sustained and that, if such motion is induced, sliding can occur even at very low levels of applied shearing tractions.

IJSS Vol. 14. No +A 


\section{FORMULATION}

The two semi-infinite solids are forced together by the applied pressure $p^{x}$ and also subjected to uniform shearing tractions $q^{\infty}$ at infinity, as indicated in Fig. 1. The separation zone propagates to the right with the velocity $c$. We assume that some specified pressure $p_{0}(\eta)$ exists inside the separation zone $\alpha<\eta<\beta$, where

$$
\eta=x-c t
$$

is the moving coordinate. The assumed pressure inside the separation zone may be due to the presence of a fluid. Although the problem is formulated in general terms, only the case of $p_{0}(\eta)=$ const will be discussed in detail.

The gap $g(\eta)$ and the relative shift $h(\eta)$ between the two solids are in terms of the displacement components

$$
g(\eta)=\left(\bar{u}_{y}-u_{y}\right)_{y=0}, \quad h(\eta)=\left(\bar{u}_{x}-u_{x}\right)_{y=0}
$$

where bars are used to refer to the upper solid. We want to have

$$
\begin{array}{lll}
g(\eta)=0, & h(\eta)=b_{x}>0 & \text { for } \eta<\alpha \\
g(\eta)>0, & & \text { for } \alpha<\eta<\beta \\
g(\eta)=0, & h(\eta)=0 & \text { for } \beta<\eta
\end{array}
$$

where $b_{x}$ is the shift that is left behind by the propagating separation zone or the total Burgers vector. We also insist that the normal interface tractions not be tensile. The gap and the shift may be viewed as being caused by distributions of moving edge dislocations inside the separation zone $\alpha<\eta<\beta$. If $B_{1}(\eta)$ and $B_{2}(\eta)$ denote the densities of the climb and glide dislocation distributions, we have

$$
g(\eta)=-\int_{\alpha}^{\eta} B_{1}(\xi) \mathrm{d} \xi, \quad h(\eta)=-\int_{\beta}^{\eta} B_{2}(\xi) \mathrm{d} \xi
$$

It follows from (3) that

$$
\int_{\alpha}^{\beta} B_{1}(\xi) \mathrm{d} \xi=b_{y}=0, \quad \int_{\alpha}^{\rho} B_{2}(\xi) \mathrm{d} \xi=b_{x}>0 .
$$

Subsonic case $\left(\mathrm{c}<\mathrm{c}_{\mathrm{T}}\right)$

Suppose that a discrete dislocation with the Burgers vector $b_{x}$ moves by glide along the $x$-axis with the constant velocity $c$. In the subsonic range, the stress components transmitted by

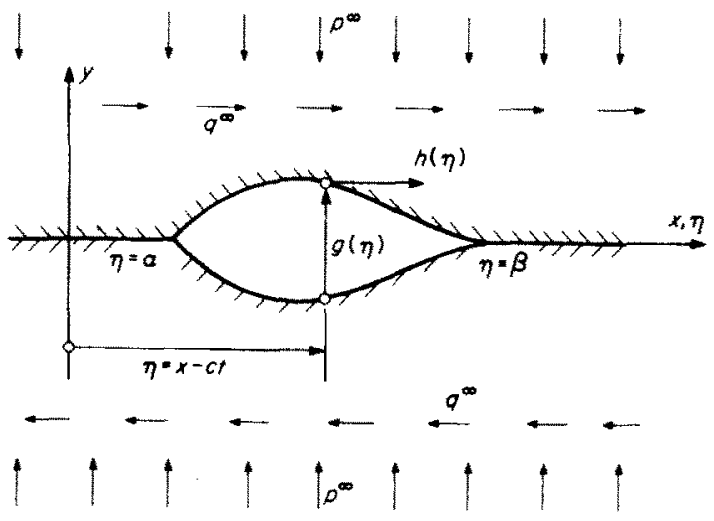

Fig. 1. 
the glide plane $y=0$ are [5]

$$
\sigma_{x y}(\eta)=-\frac{\mu b_{x} c_{T}^{2} R}{2 \pi c^{2} \zeta_{2}} \frac{1}{\eta}, \quad \sigma_{y y}(\eta)=0
$$

The Rayleigh polynomial

$$
R=\left(1+\zeta_{2}^{2}\right)^{2}-4 \zeta_{1} \zeta_{2}
$$

where

$$
\zeta_{1}=\left(1-c^{2} / c_{L}^{2}\right)^{1 / 2}, \quad \zeta_{2}=\left(1-c^{2} / c_{T}^{2}\right)^{1 / 2}
$$

and $c_{L}$ and $c_{T}$ are the speeds of longitudinal and transverse waves. Similarly, for a discrete dislocation with the Burgers vector $b_{y}$ moving by climb,

$$
\sigma_{x y}(\eta)=0, \quad \sigma_{y y}(\eta)=-\frac{\mu b_{y} c_{T}^{2} R}{2 \pi c^{2} \zeta_{1}} \frac{1}{\eta}
$$

on $y=0$.

If we think of the separation zone $\alpha<\eta<\beta$ as an interval containing moving dislocations with both climb and glide components, the duty of the moving distributed dislocations is to change the pressure inside the separation zone $\alpha<\eta<\beta$ from $p^{\infty}$ to $p_{0}(\eta)$ and the shearing traction from $q^{\infty}$ to 0 . Noting (6) and (9), these requirements lead immediately to the equations

$$
\begin{gathered}
-p^{\infty}-\frac{\mu c_{T}^{2} R}{2 \pi c^{2} \zeta_{1}} \int_{\alpha}^{\beta} \frac{B_{1}(\xi) \mathrm{d} \xi}{\eta-\xi}=-p_{0}(\eta), \quad \alpha<\eta<\beta \\
q^{\infty}-\frac{\mu c_{T}^{2} R}{2 \pi c^{2} \zeta_{2}} \int_{\alpha}^{\beta} \frac{B_{2}(\xi) \mathrm{d} \xi}{\eta-\xi}=0, \quad \alpha<\eta<\beta
\end{gathered}
$$

with the side conditions (5).

\section{Supersonic case $\left(\mathrm{c}_{\mathrm{L}}<\mathrm{c}\right)$}

For the supersonic case, Weertman[5] gives directly the interface tractions for a climb distribution $B_{1}(\eta)$ as

$$
\begin{aligned}
& \sigma_{x y}(\eta)=0 \\
& \sigma_{y y}(\eta)=-\frac{2 \mu B_{1}(\eta)}{\zeta_{2}^{2}+1}\left[\zeta \frac{1}{2}+\frac{\left(\zeta 2^{2}-1\right)^{2}}{4 \zeta_{1}^{*}}\right]
\end{aligned}
$$

and for a glide distribution $B_{2}(\eta)$ as

$$
\begin{aligned}
& \sigma_{x y}(\eta)=-\frac{2 \mu B_{2}(\eta)}{\zeta^{* 2}+1}\left[\zeta_{1}^{*}+\frac{\left(\zeta_{2}^{2}-1\right)^{2}}{4 \zeta_{2}^{*}}\right] \\
& \sigma_{y y}(\eta)=0
\end{aligned}
$$

where

$$
\zeta_{1}^{*}=\left(c^{2} / c_{L}^{2}-1\right)^{1 / 2}, \quad \zeta_{2}^{*}=\left(c^{2} / c_{T}^{2}-1\right)^{1 / 2} .
$$

Note that the tractions are completely determined by the local values of the dislocation densities.

Using (13) and (14), the conditions on the interface tractions inside the separation zone 
become

$$
\begin{gathered}
-p^{\infty}-\frac{2 \mu B_{1}(\eta)}{\zeta \psi^{2}+1}\left[\zeta \frac{\zeta}{2}+\frac{\left(\zeta 2^{2}-1\right)^{2}}{4 \zeta_{1}^{*}}\right]=-p_{0}(\eta), \quad \alpha<\eta<\beta \\
q^{\infty}-\frac{2 \mu B_{2}(\eta)}{\zeta 2^{2}+1}\left[\zeta \frac{\left(\zeta \xi^{2}-1\right)^{2}}{4 \zeta \zeta_{2}^{*}}\right]-0, \quad \alpha<\eta<\beta .
\end{gathered}
$$

The first of the conditions (5) that the total climb Burgers vector vanishes, yields if combined with (15),

$$
(\beta-\alpha) p^{\infty}=\int_{\alpha}^{\beta} p_{0}(\xi) \mathrm{d} \xi
$$

This requirement is satisfied if $p_{0}(\eta)$ is uniform and equal to $p^{\infty}$, but then $B_{1}(\eta)$ vanishes and there is no gap. Thus $p_{0}(\eta)$ cannot be constant, though it must have an average value equal to $p^{*}$. Since we discuss only the possibility of $p_{0}(\eta)=$ const, the supersonic case will not be considered further in the present article.

\section{Transonic case $\left(\mathrm{c}_{\mathrm{T}}<\mathrm{c}<\mathrm{c}_{\mathrm{V}}\right)$}

In the case of transonic motion, Weertman[5] gives the interface tractions for a climb distribution $B_{1}(\eta)$ as

$$
\begin{aligned}
& \sigma_{x y}(\eta)=0 \\
& \sigma_{y y}(\eta)=\mu\left\{-C_{1}^{*} B_{1}(\eta)+\frac{C_{1}}{\pi} \int_{\alpha}^{\beta} \frac{B_{1}(\xi) \mathrm{d} \xi}{\eta-\xi}\right\}
\end{aligned}
$$

and for a glide distribution $B_{2}(\eta)$ as

$$
\begin{aligned}
& \sigma_{y y}(\eta)=0 \\
& \sigma_{x y}(\eta)=\mu\left\{-C_{2} B_{2}(\eta)+\frac{C_{2}}{\pi} \int_{\alpha}^{\beta} \frac{B_{2}(\xi) \mathrm{d} \xi}{\eta-\xi}\right\} .
\end{aligned}
$$

Here

$$
\begin{array}{ll}
C_{1}=-\frac{2 c_{T}^{2} \zeta^{4}}{c^{2} \zeta_{1}} & C_{1}^{*}=\frac{2 c_{T}^{2} \zeta_{2}^{*}}{c^{2}} \\
C_{2}=\frac{2 c_{T}^{2} \zeta_{1}}{c^{2}} & C_{2}^{*}=\frac{2 c_{T}^{2} \zeta^{4}}{c^{2} \zeta_{2}^{*}}
\end{array}
$$

and

$$
\zeta=\left(1-c^{2} / 2 c_{T}^{2}\right)^{1 / 2}
$$

The conditions on the interface tractions yield for this case

$$
\begin{aligned}
-p^{\infty}+\mu\left\{-C_{1}^{*} B_{1}(\eta)+\frac{C_{1}}{\pi} \int_{\alpha}^{\beta} \frac{B_{1}(\xi) \mathrm{d} \xi}{\eta-\xi}\right\}=-p_{0}(\eta), & \alpha<\eta<\beta \\
q^{\infty}+\mu\left\{-C_{2}^{*} B_{2}(\eta)+\frac{C_{2}}{\pi} \int_{\alpha}^{\beta} \frac{B_{2}(\xi) \mathrm{d} \xi}{\eta-\xi}\right\}=0, & \alpha<\eta<\beta .
\end{aligned}
$$

In addition, the side conditions (5) are applicable.

The subsonic and transonic cases are studied separately in the subsequent sections. 


\section{SUBSONIC MOTION}

The interval $(\alpha, \beta)$ is the normalized by the following change of variables:

$$
\eta=\frac{1}{2}(\beta-\alpha) s+\frac{1}{2}(\beta+\alpha), \quad \xi=\frac{1}{2}(\beta-\alpha) u+\frac{1}{2}(\beta+\alpha) .
$$

In terms of the new variables (10), (11) and (5) become

$$
\begin{gathered}
\frac{a}{\pi} \int_{-1}^{1} \frac{B_{1}(u) \mathrm{d} u}{u-s}=p^{\infty}-p_{0}(s), \quad|s|<1 \\
\frac{b}{\pi} \int_{-1}^{1} \frac{B_{2}(u) \mathrm{d} u}{u-s}=-q^{\infty} \quad|s|<1 \\
\int_{-1}^{1} B_{1}(u) \mathrm{d} u=0 \\
\int_{-1}^{1} B_{2}(u) \mathrm{d} u=\frac{2 b_{x}}{\beta-\alpha}>0
\end{gathered}
$$

where

$$
a=\frac{\mu c_{I}^{2} R}{2 c^{2} \zeta_{1}}, \quad b=\frac{\mu c_{T}^{2} R}{2 c^{2} \zeta_{2}} .
$$

The solutions of the Cauchy singular integral eqns (25) and (26) are known [6, Section 113], and they depend on whether integrable singularities are admitted at any of the ends of the interval $(-1,1)$. For solutions bounded at both ends, the right sides of $(25)$ and (26) must satisfy certain consistency conditions. For (25) this condition is

$$
\int_{-1}^{1} \frac{\left[p^{\infty}-p_{0}(u)\right] \mathrm{d} u}{\left(1-u^{2}\right)^{1 / 2}}=0
$$

and $p_{0}(\eta)$ would have to be larger than $p^{\infty}$ over part of the separation zone. Since we consider only $p_{0}(\eta)=p_{0}=$ const, and $p_{0}=p^{\infty}$ gives $B_{1}(\eta)=0$, the consistency condition cannol be satisfied. Similarly, (26) has no bounded solution. Thus $B_{1}(\eta)$ and $B_{2}(\eta)$ must have at least one integrable singularity. However, the side condition (27) imposes a further restriction on $B_{1}(\eta)$. It can be shown using the general results[6, Section 113] that no solutions of (25) that are singular at one end only and satisfy (27) exist, but the details are omitted here.

The solution of (25) singular at both ends of the separation zone is

$$
B_{1}(s)=\left(1-s^{2}\right)^{m-1 / 2}\left\{-\frac{p^{\infty}-p_{0}}{\pi a} \int_{-1}^{1} \frac{\left(1-u^{2}\right)^{1 / 2} \mathrm{~d} u}{u-s}+C\right\}, \quad|s|<1 .
$$

The constant $C$ is determined from (27) to be zero. Performing the clementary integrations in (31), we obtain

$$
B_{1}(s)=\frac{p^{\infty}-p_{0}}{a} \frac{s}{\left(1-s^{2}\right)^{1 / 2}}, \quad|s|<1
$$

From (9), the total normal traction outside the gap is in terms of the new variables

$$
N(s)=-p^{\infty}+\frac{a}{\pi} \int_{-1}^{1} \frac{B_{1}(u) \mathrm{d} u}{u-s}, \quad|s|>1 .
$$


Substituting (32) and integrating,

$$
N(s)=-p_{0}-\left(p^{\infty}-p_{0}\right) \frac{|s|}{\left(s^{2}-1\right)^{1 / 2}}, \quad|s|>1
$$

and for the interface tractions to be compressive, we must have

$$
p_{0}<p^{\infty}
$$

Finally, the gap from (4) is in terms of the new variables

$$
\begin{aligned}
g(s)=-\frac{1}{2}(\beta-\alpha) \int_{-1}^{s} B_{1}(u) \mathrm{d} u & =\frac{\left(p^{\infty}-p_{0}\right)(\beta-\alpha) c^{2} \zeta_{1}}{\mu c_{T}^{2} R}\left(1-s^{2}\right)^{1 / 2}, \\
|s| & <1 .
\end{aligned}
$$

To avoid interpenetration, the gap must be nonnegative. 'This requirement imposed on (36) yields

$$
R>0, \text { or } c>c_{R}
$$

where $c_{R}$ is the Rayleigh wave velocity of the solids. The situation is thus analogous to the propagation of interface waves involving separation[7]. It should be noted that the normal interface tractions do not depend on the propagation velocity of the separation zone.

Turning now to the distribution of the glide dislocations, we must consider three possibilities:

Shearing tractions singular at the trailing edge of the separation zone

In this case [6, Section 113]

$$
B_{2}(s)=\frac{q^{\infty}}{\pi b}\left(\frac{1-s}{1+s}\right)^{1 / 2} \int_{-1}^{1}\left(\frac{1+u}{1-u}\right)^{1 / 2} \frac{\mathrm{d} u}{u-s}=\frac{q^{\infty}}{b}\left(\frac{1-s}{1+s}\right)^{1 / 2}, \quad|s|<1
$$

The tangential shift or the total Burgers vector of the glide distribution is computed from (28):

$$
b_{x}=\frac{\pi q^{\infty}(\beta-\alpha)}{2 b}=\frac{\pi q^{\infty} c^{2} \zeta_{2}(\beta-\alpha)}{\mu c_{T}^{2} R}
$$

For $c>c_{R}$, the shift occurs in the direction of the gap propagation. The shearing tractions outside the gap are given by

$$
T(s)=q^{\infty}+\frac{b}{\pi} \int_{-1}^{1} \frac{B_{2}(u) \mathrm{d} u}{u-s}, \quad|s|>1 .
$$

Inserting (38) into (40),

$$
T(s)=q^{\infty}\left|\frac{s-1}{s+1}\right|^{1 / 2}, \quad|s|>1
$$

Shearing tractions singular at the leading edge of the separation zone

Following the same procedure as in the previous case, we obtain

$$
\begin{aligned}
B_{2}(s) & =\frac{q^{\infty}}{\pi b}\left(\frac{1+s}{1-s}\right)^{1 / 2} \int_{-1}^{1}\left(\frac{1-u}{1+u}\right)^{1 / 2} \frac{\mathrm{d} u}{u-s} \\
& =-\frac{q^{\infty}}{b}\left(\frac{1+s}{1-s}\right)^{1 / 2}, \quad|s|<1
\end{aligned}
$$




$$
\begin{gathered}
b_{x}=-\frac{\pi q^{\infty} c^{2} \zeta_{2}(\beta-\alpha)}{\mu c_{T}^{2} R} \\
T(s)=q^{\infty}\left|\frac{s+1}{s-1}\right|^{1 / 2}, \quad|s|>1 .
\end{gathered}
$$

It is noted that the tangential shift is now in the direction opposite to the gap propagation. This is contrary to our requirement (5), and the case will not be considered further.

\section{Shearing tractions singular at both edges of the separation zone}

The distribution $B_{2}(s)$ of the glide dislocations is of the form (31) with $\left(p^{\infty}-p_{0}\right) / a$ replaced by $-q^{\infty} / b$, and we obtain

$$
B_{2}(s)=\left(1-s^{2}\right)^{-1 / 2}\left(C-q^{\infty} s / b\right), \quad|s|<1 .
$$

The constant $C$ is determined from (28) as

$$
C=\frac{2 b_{x}}{\pi(\beta-\alpha)}
$$

The shearing tractions at the interface are from (40)

$$
T(s)=\left(s^{2}-1\right)^{-1 / 2}\left(q^{\alpha}|s|-C b \operatorname{sgn} s\right), \quad|s|>1 .
$$

In the contact regions, the shearing tractions must not exceed in magnitude the maximum static friction if slip is not to take place. Thus

$$
|T(\eta)|<f_{s}|N(\eta)|
$$

where $f_{s}$ denotes the coefficient of static friction.

Returning to the original variable $\eta$, the normal traction is from (34)

$$
\begin{gathered}
N(\eta)=-p_{0}-\left(p^{\infty}-p_{0}\right) \frac{\left|\eta-\frac{\beta+\alpha}{2}\right|}{[(\eta-\beta)(\eta-\alpha)]^{1 / 2}}, \\
\eta<\alpha \text { and } \beta<\eta .
\end{gathered}
$$

For singularity in the shearing tractions at the trailing edge only,

$$
T(\eta)=q^{\infty}\left|\frac{\eta-\beta}{\eta-\alpha}\right|^{1 / 2}, \quad \eta<\alpha \text { and } \beta<\eta .
$$

The inequality (48) is most critical at $\eta=\alpha$, and it yields the condition

$$
q^{\infty}<\frac{1}{2} f_{s}\left(p^{\infty}-p_{0}\right)
$$

If the shearing tractions are singular at both edges of the separation zone, (48) leads to

$$
q^{\infty}<\left(f_{s}-\frac{b_{x} \mu c_{T}^{2} R}{\pi c^{2} \zeta_{2}(\beta-\alpha)}\right)\left(p^{\infty}-p_{0}\right)
$$

In both cases, sliding can take place with much lower applied shearing tractions than required for frictional slip. 
Normalizing the separation interval $(\alpha, \beta)$ by means of (24) and assuming constant internal pressure, (22) and (23) become

$$
\begin{array}{ll}
C_{1}^{*} B_{1}(s)+\frac{C_{1}}{\pi} \int_{-1}^{1} \frac{B_{1}(u) \mathrm{d} u}{u-s}=-\frac{p^{*}-p_{0}}{\mu}, & |s|<1 \\
C_{2}^{*} B_{2}(s)+\frac{C_{2}}{\pi} \int_{-1}^{1} \frac{B_{2}(u) \mathrm{d} u}{u-s}=\frac{q^{\infty}}{\mu}, & |s|<1,
\end{array}
$$

The side conditions (27) and (28) remain in force.

The Cauchy singular integral equations are now of the second kind. Their solution is known [6, Section 107]. The corresponding consistency conditions cannot be satisfied if the right sides of (53) and (54) are constant, and there are no bounded solutions.

The general solution of (53) is

$$
B_{1}(s)=-\frac{p^{*}-0_{0}}{\mu\left(C_{1}^{2} \mid C^{* 2}\right)}\left\{C_{1}^{*}-\frac{1}{\pi} C_{1} w(s) \int_{-1}^{1} \frac{\mathrm{d} u}{w(u)(u-s)}\right\}+C w(s),|s|<1
$$

where $w(s)$ is the characteristic function, and the constant $C$ may not be zero only for solutions singular at both end points. The characteristic function depends on the singularities admitted. As in the subsonic case, there are no solutions for $B_{1}(\eta)$ that are singular only at one end of the interval and satisfy $(27)$. Thus $B_{1}(\eta)$ must be singular at both ends, and the characteristic function is

$$
w(s)=(1-s)^{A-1}(1+s)^{-A}
$$

where

$$
\tan \pi A=-\frac{C_{1}}{C_{1}^{*}}=\frac{\zeta^{4}}{\zeta_{1} \zeta_{2}^{*}}, \quad 0<A<1
$$

Applying (27), the constant $C$ in (55) is determined to be zero. The integrals in (55) can be found in tables [8. No. 3.196 and 3.228]. and thus

$$
B_{1}(s)=\frac{p^{x}-p_{0}}{\mu\left(C_{1}^{2}+C_{1}^{* 2}\right)^{1 / 2}}(1-s)^{A-1}(1+s)^{-A}(2 A-1+s), \quad|s|<1
$$

The normal tractions in the contact zones are

$$
N(s)=-p^{\infty}-\frac{\mu C_{1}}{\pi} \int_{-1}^{1} \frac{B_{1}(u) \mathrm{d} u}{u-s}=-p_{0}-\left(p^{\infty}-p_{0}\right)|1-s|^{A-1}|1+s|^{-A}|2 A-1+s|, \quad|s|>1 .
$$

Finally, from (4)

$$
\frac{\mathrm{d} g(\eta)}{\mathrm{d} \eta}=-B_{1}(\eta)
$$

and it can be shown that the gap is positive.

The solution of (54) is obviously of the same form as (55); only $-\left(p^{\infty}-p_{0}\right) / \mu, C_{1}, C_{1}^{*}$ need be replaced by $q^{\star} / \mu, C_{2}, C_{2}^{*}$. We distinguish again three cases for $B_{2}(\eta)$ :

Singularity at the trailing edge

The characteristic function is then

$$
w(s)=\left(\frac{1-s}{1+s}\right)^{B}
$$


where

$$
\tan \pi B=-\frac{C_{2}}{C_{2}^{*}}=-\frac{\zeta_{1} \zeta_{2}^{*}}{\zeta^{4}}, \quad 0<B<1
$$

The dislocation density and the shearing tractions are obtained as explained before, and

$$
\begin{array}{cc}
B_{2}(s)=-\frac{q^{\infty}}{\mu\left(C_{2}^{2}+C_{2}^{w^{2}}\right)^{1 / 2}}\left(\frac{1-s}{1+s}\right)^{B}, & |s|<1 \\
T(s)=q^{*}-\frac{\mu C_{2}}{\pi} \int_{-1}^{1} \frac{B_{2}(u) \mathrm{d} u}{u-s}=q^{\infty}\left|\frac{s-1}{s+1}\right|^{B}, & |s|>1 .
\end{array}
$$

From (28) and (62) we determine

$$
b_{x}=-\frac{\pi q^{*} B(\beta-\alpha)}{\mu C_{2}}<0
$$

Note that the tangential shift is now opposite to the direction in which the gap propagates. In the subsonic case, the reversal in sign occurred for singularities at the leading edge.

\section{Singularity at the leading edge}

The relevant quantities are

$$
\begin{gathered}
w(s)=\left(\frac{1+s}{1-s}\right)^{1-B} \\
B_{2}(s)=\frac{q^{*}}{\mu\left(C_{2}^{2}+C_{2}^{*^{2}}\right)^{1 / 2}}\left(\frac{1+s}{1-s}\right)^{1-\beta},|s|<1 \\
T(s)=q^{\infty x}\left|\frac{s+1}{s-1}\right|^{1-B}, \quad|s|>1 \\
b_{x}=\frac{\pi q^{\infty}(1-B)(\beta-\alpha)}{\mu C_{2}}>0 .
\end{gathered}
$$

Singularities at both edges

The results are

$$
\begin{gathered}
w(s)=(1-s)^{B-1}(1+s)^{-B} \\
B_{2}(s)=(1-s)^{B-1}(1+s)^{-B}\left\{\frac{q^{\infty}}{\mu\left(C_{2}^{2}+C_{2}^{x^{2}}\right)^{n / 2}}(2 B-1+s)+C\right\},|s|<1 \\
C=\frac{2 b_{x} \sin \pi B}{\pi(\beta-\alpha)} \\
T(s)=-\left\{q^{\infty}(2 B-1+s)+\frac{2 b_{x} \mu C_{2}}{\pi(\beta-\alpha)}\right\} \frac{1}{1-s}\left|\frac{1-s}{1+s}\right|^{B}, \quad|s|>1 .
\end{gathered}
$$

Examining (48) for the three cases in the transonic range and noting that the singularities in $N(s)$ and $T(s)$ are of different orders, it follows that the inequality can only be satisfied when the singularity is at the leading edge $\eta=\beta$ of the separation zone. It is then sufficient to require

$$
q^{\infty}<f_{s}\left(p^{\infty}-p_{0}\right)
$$

Thus sliding without slip can occur in the transonic range with less than the shearing stress 
required for frictional slipping, but only if there is a singularity in the shearing fractions at the leading edge of the separation zone.

\section{CONCLUSION}

It is clear from the integral equations, and also the other conditions imposed, that the separation between the solids and the sliding motion are only weakly coupled. The coupling appears merely through the friction law (48). A consequence of this is that the applied shearing tractions do not drive the separation between the bodies, and that such separation would have to be induced by other sources at the beginning of the motion.

It is seen from (39) that, for the subsonic case and singularity in the shearing tractions at the trailing edge, the tangential shift is determined by the applied shearing tractions, length of the separation zone and its speed of propagation. In contrast, the shift remains arbitrary if the interface sharing tractions are singular at hoth ends of the separation zone. In such case, the shift enters only the relation (52) which restricts the magnitude of the applied shearing tractions. For transonic motion, a singularity could be allowed only at the leading edge, and the shift is determined by (68).

\section{REFERENCES}

1. J. J. Gilman, Micromechanics of Flow in Solids. McGraw-Hill. New York (1969).

2. A. Schallamach, How does rubber slide? Wear 17.301 (1971).

3. K. Kendall. Model experiments illustrating fibre-pull out. J. Mater. Sci. 10, 1011 (1975)

4. M. Barquins and R. Courtel, Rubber friction and the rheology of viscoelastic contact. Wear 32, 133 (1975).

5. J. Weertman. Dislocations in uniform motion on slip or climb planes having period force laws. Mathematical Theory of Dislocations (Edited by T. Mura), pp. 178-202. The American Society of Mechanical Engineers (1969).

6. N. I. Muskhelishvili, Singular Integral Equations. P. Noordhoff, Leyden (1953).

7. M. Comninou and J. Dundurs. Elastic interface waves involving separation. J. Appl. Mech. 44. 222 (1977)

8. I. S. Gradshteyn and I. M. Ryzhik. Tuble of Integrals. Series and Products. Academic Press. New York (19651. 